Cinémas

Revue d'études cinématographiques

Journal of Film Studies

\title{
"It is man's work and you are just little girlies " : narration genrée et figures de l'empowerment féminin dans le film catastrophe hollywoodien
}

\section{François-Xavier Molia}

Volume 22, numéro 2-3, printemps 2012

Genre/Gender

URI : https://id.erudit.org/iderudit/1011656ar

DOI : https://doi.org/10.7202/1011656ar

Aller au sommaire du numéro

Éditeur(s)

Cinémas

ISSN

1181-6945 (imprimé)

1705-6500 (numérique)

Découvrir la revue

Citer cet article

Molia, F.-X. (2012). "It is man's work and you are just little girlies » : narration genrée et figures de l'empowerment féminin dans le film catastrophe hollywoodien. Cinémas, 22(2-3), 81-99. https://doi.org/10.7202/1011656ar

\section{Résumé de l'article}

Le film catastrophe hollywoodien des années 1970 reproduisait les représentations traditionnelles des rapports de sexe dans une société patriarcale. Le cycle des années 1990 semble, lui, prendre acte des évolutions de la société contemporaine en accordant aux femmes une présence filmique accrue ainsi que des statuts sociaux plus importants. À quelle éventuelle élaboration générique ce rééquilibrage de la galerie des personnages conduit-il ? Twister (Jan de Bont, 1996), Deep Impact (Mimi Leder, 1998) et Titanic (James Cameron, 1997) modifient la formule du film catastrophe en y greffant des éléments susceptibles (du moins le suppose-t-on) d'attirer les spectatrices vers un genre " pour hommes " : traitement approfondi des enjeux amoureux et familiaux, hybridation avec le mélodrame ou la romance et amplification de la tonalité pathétique et, enfin, travail sur le point de vue féminin à travers différents procédés narratifs. Cette réorientation produit du " texte incohérent » (Robin Wood), sur le plan générique et sur le plan des représentations genrées, puisque ces femmes agissantes mais malgré tout dominées font osciller les films entre vision progressiste et stéréotypes. 


\title{
"It is man's work and you are just little girlies»: narration genrée et figures de l'empowerment féminin dans le film catastrophe hollywoodien
}

\section{François-Xavier Molia}

\begin{abstract}
RÉSUMÉ
Le film catastrophe hollywoodien des années 1970 reproduisait les représentations traditionnelles des rapports de sexe dans une société patriarcale. Le cycle des années 1990 semble, lui, prendre acte des évolutions de la société contemporaine en accordant aux femmes une présence filmique accrue ainsi que des statuts sociaux plus importants. À quelle éventuelle élaboration générique ce rééquilibrage de la galerie des personnages conduit-il? Twister (Jan de Bont, 1996), Deep Impact (Mimi Leder, 1998) et Titanic (James Cameron, 1997) modifient la formule du film catastrophe en y greffant des éléments susceptibles (du moins le suppose-t-on) d'attirer les spectatrices vers un genre "pour hommes": traitement approfondi des enjeux amoureux et familiaux, hybridation avec le mélodrame ou la romance et amplification de la tonalité pathétique et, enfin, travail sur le point de vue féminin à travers différents procédés narratifs. Cette réorientation produit du "texte incohérent» (Robin Wood), sur le plan générique et sur le plan des représentations genrées, puisque ces femmes agissantes mais malgré tout dominées font osciller les films entre vision progressiste et stéréotypes.
\end{abstract}

Une femme au bord des larmes, à qui la peur tire des cris suraigus, se blottit contre le torse de son protecteur masculin: l'image court de The Poseidon Adventure (Ronald Neame, 1972) à Volcano (Mick Jackson, 1997), en passant par The Towering Inferno (John Guillermin, 1974), Earthquake (Mark Robson, 1974), Twister (Jan de Bont, 1996)... Saturés de représentations patriarcales, les films catastrophe ont le plus souvent été analysés 
comme des œuvres réactionnaires, dont les intrigues proposent des solutions conservatrices à des crises collectives, à travers la réaffirmation de la puissance et de la légitimité masculines ${ }^{1}$. Très largement recevable, cette lecture fait cependant l'impasse sur les ambivalences du genre. À propos de Jaws (Steven Spielberg, 1975), un critique de cinéma relevait ce paradoxe: "Les peurs, comme celles que suscitent The Poseidon Adventure et The Towering Inferno, ne sont pas écartées par les dénouements heureux" (Champlin 1975, p. 35) ${ }^{2}$. Autrement dit, la charge anxiogène d'un film peut persister en dépit du retour à l'ordre final. La remarque du critique nous invite à réviser la perception des effets filmiques telle que la pensée critique d'inspiration marxiste a pu les appréhender: les prescriptions idéologiques ne sont pas forcément le dernier mot d'un film; elles peuvent être brouillées, contredites, concurrencées par d'autres éléments et d'autres processus à l'œuvre. En outre, comme l'indique Raphaëlle Moine (2010, p. 49-51) dans Les femmes d'action au cinéma, discours réactionnaire et progressiste peuvent se trouver étroitement mêlés à l'intérieur d'un même film, dans une industrie qui a fait de la contradiction un principe esthétique lui permettant de toucher des publics très hétérogènes. Enfin, selon l'hypothèse interprétative que l'on formule, un même élément peut être investi de significations radicalement divergentes. L'attribution de caractères virils à certaines héroïnes de films d'action, par exemple, est-elle une manière d'affirmer la puissance des femmes ou bien de les cantonner dans une dépendance au masculin posé comme modèle indépassable de l'affirmation de soi (p. 31)?

C'est à partir de cette attention aux contradictions et aux équivoques du discours filmique qu'une lecture genrée du film catastrophe ${ }^{3}$ pourra mettre en lumière les divergences d'un film à l'autre: car, si certains d'entre eux adoptent une position conservatrice relativement cohérente, d'autres, dans les années 1990, proposent un traitement du féminin pris entre progressisme et régression. Sur des récits célébrant l'hérö̈sme masculin viennent ainsi se greffer des images de l'autonomie ou de la puissance féminines qui dérogent aux représentations dominantes. Cependant, la mise en évidence de ces ambivalences remet en 
question plus profondément la place et l'activité du spectateur. Car ce sont ses préférences interprétatives qui détermineront sans doute le positionnement idéologique du film dans sa dimension gender.

\section{Femmes désastreuses}

Le film catastrophe hollywoodien des années 1970 reproduit globalement les représentations dominantes des rapports de sexe dans le cinéma classique hollywoodien. À ce titre, le genre se démarque très nettement d'un certain nombre de films qui, à la même époque, interrogent les normes sexuées dans le cadre d'une remise en cause des conventions esthétiques et éthiques de la tradition hollywoodienne: on pense aussi bien ici à certains woman's films comme Wanda (Barbara Loden, 1970) et Alice Doesn't Live Here Anymore (Martin Scorsese, 1974), qu'aux dystopies science-fictionnelles comme The Stepford Wives (Bryan Forbes, 1975) et Alien (Ridley Scott, 1979). Le film catastrophe fut ainsi largement perçu, dès les années 1970, comme un genre conservateur, participant du changement de climat social qui devait mener au cinéma dit « reaganien ${ }^{4}$ ».

Conformément à la perception dominante de la féminité dans une société patriarcale, les personnages féminins y sont associés à la passivité et à la faiblesse. Dans The Poseidon Adventure, la mise en scène souligne le manque d'autonomie des quatre femmes du groupe de survivants et les renvoie dans une forme de minorité symbolique par rapport aux personnages masculins. Les femmes sont régulièrement figurées en train de les suivre, de s'appuyer sur leurs bras ou d'y blottir leur visage effrayé. Très souvent désemparées et en larmes, elles ne trouvent la force de continuer que dans le refus du renoncement auquel les encouragent les hommes. Elles ont besoin de se soumettre au leadership masculin pour surmonter les épreuves.

Le film le plus célèbre du cycle des années 1970, The Towering Inferno, porte par ailleurs un regard particulièrement sévère sur les femmes, puisque son scénario les rend responsables de l'échec d'une tentative de sauvetage par le toit du gratte-ciel. En s'y précipitant de manière désordonnée, elles obligent l'hélicoptère à manœuvrer brusquement pour ne pas les blesser, ce 


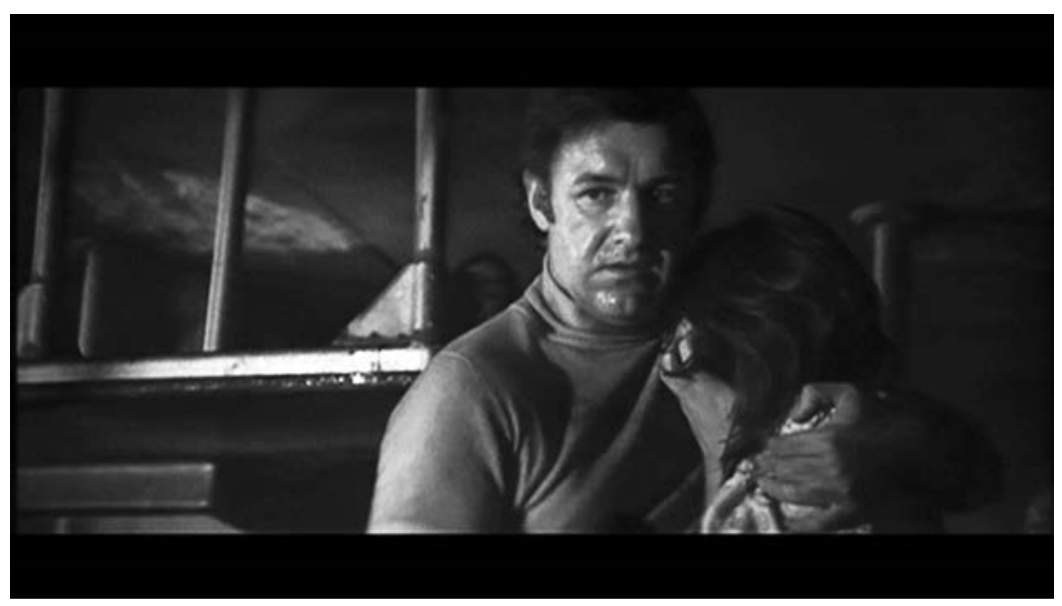

The Poseidon Adventure (Ronald Neame, 1972). ( ) Twentieth Century Fox.

qui conduit l'engin au crash et à l'explosion. Les femmes sont, ici, littéralement désastreuses.

Marqué par l'hystérie, le corps féminin apparaît en outre difficilement contrôlable: il faut parfois le gifler, comme dans The Poseidon Adventure, pour l'apaiser un peu. Dans ce film, il est significatif que l'identité de la vieille Belle (Shelley Winters) soit implicitement virilisée lorsqu' elle se porte au secours du révérend Scott (Gene Hackman) en l'aidant à se libérer d'une lourde porte qui le coince sous l'eau. Le personnage de Rogo (interprété par Ernest Borgnine, spécialiste des rôles de durs à cuire) se penche sur Belle et murmure: "You had a lot of guts, lady. A lot of guts" ("Vous avez eu de sacrées tripes, madame, de sacrées tripes»). Le mot guts renvoie à l'univers viril du western, tandis que l'identité sexuelle de Belle est rappelée par lady. Tout se passe donc comme si la prouesse sous-marine de la vieille dame lui avait permis d'accéder à l'hérö̈que en même temps qu'au masculin. (Il est à noter que cet exploit, devenir un homme, lui est cependant fatal: elle succombe sitôt après d'un arrêt cardiaque.)

\section{«Boulots d'hommes"}

Comme on peut s'y attendre, les films catastrophe des années 1990 reflètent au moins en partie les changements intervenus au cours des dernières décennies dans les sociétés occidentales et font état d'une promotion sociale des femmes à travers le 
type d'emploi qu'elles occupent: dans Volcano, Anne Heche joue une scientifique de terrain à l'esprit indépendant; dans Deep Impact (Mimi Leder, 1998), Téa Leoni incarne une ambitieuse journaliste; Linda Hamilton est la mairesse courageuse d'une petite commune de montagne dans Dante's Peak (Roger Donaldson, 1997), et le conseil municipal qu'elle dirige est pour moitié féminin. Le scénario de ce film témoigne, semble-t-il, de l'accès des femmes à des postes de responsabilité dans la société civile, et plus particulièrement de la féminisation de la vie politique américaine. Enfin, dans Titanic (James Cameron, 1997) et Armageddon (Michael Bay, 1998), Rose (Kate Winslet) et Grace (Liv Tyler) sont des jeunes femmes encore sans profession, mais qui revendiquent le droit à l'autonomie face à un ordre patriarcal rigide. De la sorte, vient se glisser dans le récit de ces différents films catastrophe un enjeu qui renvoie directement à la condition sociale des femmes et au woman's film: leur capacité à réussir professionnellement, à s'insérer dans un espace dont elles ont traditionnellement été exclues ou encore à s'épanouir individuellement contre la volonté de leur milieu.

Ce rééquilibrage du "personnel fictionnel» conduit-il à une recomposition d'un genre jusqu'ici marqué par la figure centrale du sauveur viril ? L'approche gender fait apparaître un problème idéologique inhérent au film catastrophe des années 1990 (l'intrusion de femmes comme protagonistes dans un genre célébrant la puissance du masculin) ainsi qu'un spectre de réponses narratives qui vont de la réaffirmation patriarcale pure et dure à quelques tentatives visant à construire un récit au féminin ${ }^{5}$. Pourra-t-on en conclure qu'il existe, au sein du genre, un courant conservateur et un courant progressiste, des «films catastrophe de droite» et des "films catastrophe de gauche »?

Dans Volcano et Dante's Peak, la mise en valeur socioprofessionnelle des femmes et la place qui leur est dévolue en tant que protagonistes n'aboutissent cependant pas à la remise en cause des normes sexuées traditionnellement véhiculées par le genre. C’est même tout le contraire: l'indépendance affichée au début du récit par les personnages féminins se résout en une subordination volontaire au pouvoir masculin, seul à même d'affronter la situation de crise créée par le désastre. Ces deux films 
catastrophe s'offrent en ce sens comme des récits de domestication d'une féminité inquiétante.

Cette contradiction manifeste entre l'émancipation superficielle de la femme moderne et sa dépendance réelle au pouvoir masculin est particulièrement sensible dans Volcano, le film de Mick Jackson. En rejouant la "bataille des sexes" chère à la comédie romantique de la période classique, le scénario oppose et apparie Mike Roark (Tommy Lee Jones) et Amy Barnes (Anne Heche). Le premier est en charge de la sécurité civile de Los Angeles. La seconde est une sismologue caractérisée par un tempérament intrépide et indocile. Le plus intéressant est qu'Amy Barnes paraît elle-même consciente des effets néfastes de la domination masculine. Lorsque Roark lui interdit de descendre dans le souterrain où a eu lieu une explosion d'origine inconnue, elle rapporte, dépitée, les propos de l'homme à sa collègue: "C'est un boulot d'homme et vous n'êtes que des fillettes. Je ne peux pas vous laisser descendre ${ }^{6}$. " Elle en conclut que, bien que sympathique, Mike Roark est un "macho». La suite du film s'efforce, superficiellement, de développer le personnage à partir de cette présentation initiale d'une jeune femme "moderne", qui n'a pas froid aux yeux et affirme son autonomie: Amy prodigue nombre de conseils d'experte et prend part à certaines actions physiques, comme lorsqu'elle aide Roark et d'autres hommes à pousser un bus en travers d'une avenue pour faire barrage à la coulée de lave. Mais ce comportement est en réalité limité par les contraintes hollywoodiennes d'un héroïsme presque exclusivement réservé aux personnages masculins. Roark sauve la vie d'Amy à deux reprises, chaque fois en la couvrant de son corps, pour qu'elle échappe à la chute d'un pylône en feu puis à une remontée de lave dans une bouche d'égout. C'est la posture iconique du masculin protégeant le féminin qui se trouve ici remise en valeur. La femme active est ainsi et malgré tout supplantée par l'homme d'action, qui est le seul véritable sauveur de la communauté. Cette relégation est cruellement évidente à la fin du film, lorsque, sans nouvelles de sa fille, Roark demande à Amy de la retrouver. On voit celle-ci s'époumoner en vain et courir en tous sens. Pour la première et unique fois du film, elle est mise en position de sau- 
ver quelqu'un... mais elle n'y parvient pas. C'est Roark luimême qui finit par apercevoir sa fille, au pied d'un immeuble promis à une imminente explosion, et qui court pour la sauver au dernier moment, dans un ralenti «héroïsant».

Un mot, également, sur la manière dont Dante's Peak utilise la persona de l'actrice Linda Hamilton. Celle-ci est surtout connue pour son interprétation de Sarah Connor, dans la série Terminator. Or Sarah Connor a subi une spectaculaire métamorphose entre le premier et le second épisode de la saga, passant du stéréotype de la jeune femme démunie à celui de la "macho woman with gun ${ }^{7}$ ", de la victime en fuite à la rebelle en marge. Au terme de cette transformation, Linda Hamilton est devenue, comme Sigourney Weaver, une icône dans la culture lesbienne anglo-saxonne ("a cult lesbian favourite», dit un blog aujourd'hui ${ }^{8}$ ). D'un côté, on peut considérer que Dante's Peak réactive, en choisissant cette actrice, le personnage de la femme indépendante et puissante: Rachel Wando, en plus de détenir le pouvoir politique au sein de sa communauté, élève seule ses deux enfants, loin d'un ex-mari présenté comme un mauvais père, démissionnaire et absent. Mais il me semble que le film opère également un infléchissement régressif par rapport à la persona de Linda Hamilton, en associant à l'actrice les attributs d'une féminité on ne peut plus traditionnelle: mère nourricière en tailleur, soucieuse de son apparence, et scrutant le miroir d'un œil inquiet, dans sa première scène, réduite par la suite au rôle passif de love interest à protéger, le personnage de Rachel réintègre, à la fin du film, le cadre normatif de la conjugalité hétérosexuelle. Harry (le héros, joué par Pierce Brosnan) retrouve Rachel et l'embrasse, sous les applaudissements des sauveteurs et devant ses deux enfants à elle, dont les rires ont valeur d'assentiment. Harry les attire d'ailleurs à lui pour former, dans une large étreinte, un noyau familial recomposé. La dernière scène, dans l'hélicoptère, apprend au spectateur que tous les quatre partent en Floride pour une partie de pêche, et un gros plan sur les mains serrées de Harry et de Rachel parachève leur union. Exit l'agressive et peu orthodoxe Sarah Connor. Linda Hamilton est redevenue une femme comme les autres. 


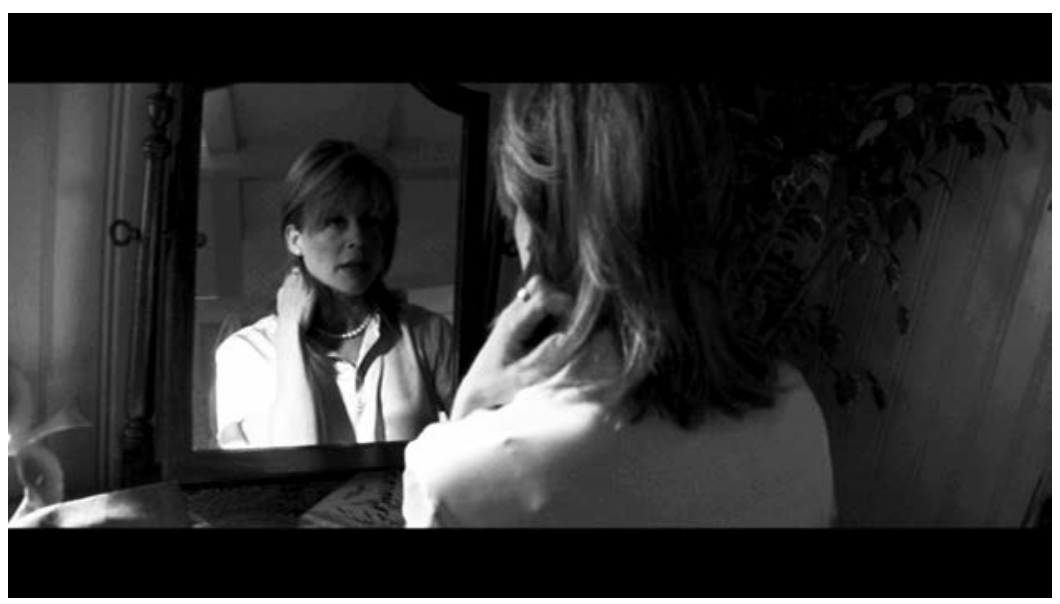

Dante's Peak (Roger Donaldson, 1997). (C) Universal Pictures.

\section{Films catastrophe au féminin}

Dans Twister, Deep Impact et Titanic, l'importance prise par les personnages féminins modifie la formule du film catastrophe en y introduisant ces éléments caractéristiques de la romance ou du mélodrame que constituent le traitement approfondi des enjeux amoureux et familiaux, l'amplification de la tonalité pathétique et le travail sur le point de vue féminin à travers différents procédés narratifs. Le recours à de tels éléments correspond à la volonté des studios d'attirer le public féminin vers des genres, film d'action ou film catastrophe, dont les femmes ont tendance à se détourner ${ }^{9}$. Cette réticence se révèle problématique dans le cadre d'une production coûteuse ayant besoin, pour devenir rentable, d'attirer le public le plus large possible.

De tous ces procédés, le plus intéressant me paraît être celui qui cherche à favoriser la participation émotionnelle du spectateur aux destinées d'un personnage de femme en développant des effets de point de vue. C'est ce que j'appelle ici "récit au féminin ": même s'il s'agit de films réalisés par des hommes, et dans lesquels on pourrait aisément déceler les occurrences d'éléments appartenant à une idéologie patriarcale, Twister et Titanic construisent des effets de point de vue féminin (je prends soin de distinguer entre "effet" et point de vue réel), c'est-à-dire donnent l'impression que l'histoire se déploie à partir d'une 
femme et même grâce à elle, ce qui invite le public à s'impliquer dans le devenir fictif d'un personnage féminin, dont le statut de figure à l'écran se double de celui d'agent de figuration. La femme est en effet ici, plus qu'un personnage principal, la condition a priori du développement diégétique.

Twister dévide son intrigue à partir de l'expérience traumatisante d'une jeune fille, Jo (Helen Hunt), exposée dans le prologue du film. En incrustation apparaît la mention "Juin 1969» ("June 1969»). Une violente tornade contraint la famille de la jeune fille à se réfugier dans un abri dont la porte ferme mal. Le père de Jo essaie de toutes ses forces de la retenir, avant d'être aspiré avec elle et emporté dans les airs. Jo, juste avant, regarde des formes imprécises que le chaos de la tornade fait passer devant un hublot. Son attitude contemplative contraste avec la peur de ses parents. Deux éléments sont ainsi posés: la fascination de Jo pour les tornades et le traumatisme enfantin lié à la mort du père, qui appelle une réparation. Le dernier plan du prologue est une vue d'ensemble de la maison dévastée, sur laquelle courent, affaiblis, les cris de terreur poussés par Jo et sa mère dans le plan précédent. Cette persistance sonore suggère le caractère traumatique d'une expérience qui continue de résonner après qu'elle est survenue. Twister s'annonce, par cette séquence d'ouverture, comme un film spectaculaire et comme le portrait d'une femme marquée par les épreuves de la vie. Mais il y a davantage encore: tout au long du récit, Jo ne cesse, par sa soif de sensations fortes, d'enclencher de nouveau l'action spectaculaire du film catastrophe. Après la double tornade qui a laissé tous ses collaborateurs médusés et pantelants, Jo s'écrie: "Ce n'est pas fini, on n'a vu que le début ${ }^{10}$ ! C'est par elle, par sa témérité et son excitation, son envie d'en voir toujours plus, que le genre livre le divertissement que l'on attend de lui. Jo régit en ce sens l'offre spectaculaire proposée par le film.

Dans Titanic, c'est l'activité de remémoration d'une femme âgée, dont la voix off intervient ponctuellement dans le récit filmique, qui donne le départ du film catastrophe et qui le rend possible: sans son témoignage, l'expérience nous resterait inconnue. Les recherches sous-marines du chasseur de trésors donnent d'abord l'occasion au spectateur de découvrir ce qui reste du 
navire, baigné dans le crépuscule des fonds marins, ses parois et tout ce qu'il contient entièrement couverts d'un dépôt grisâtre. Spectaculaires, ces premières images du Titanic sont cependant placées sous le signe du manque et de la vision fragmentaire. Le sous-marin miniature téléguidé par les explorateurs éclaire une botte déchirée, des lunettes cassées, la tête d'une poupée en porcelaine et de multiples débris. Brock Lovett (Bill Paxton) croit que le bijou dont il veut s'emparer est à l'abri d'un coffre-fort mais celui-ci, une fois remonté et forcé, n’a que des papiers humides à lui offrir, trempés dans une boue couleur rouille. Ces premières séquences contemporaines font donc voir le navire en même temps qu'elles frustrent la vision: le Titanic est bien là, mais ce que l'on espère y trouver, son faste proverbial, est quant à lui absent. Après cette entrée déceptive dans la fiction, l'apparition de Rose (Gloria Stuart) joue de façon subtile sur la reprise du motif de la boue - mais cette fois en inversant sa valeur. On découvre en effet Rose en vieille potière, qui transforme l'argile rouge en vase. Ses mains donnent forme au même type de matière décevante que Brock Lovett vient de trouver en lieu et place du bijou. Le principe de narration du film est annoncé dans cette représentation: Rose est le personnage qui recrée le Titanic à partir de ses vestiges informes. Cette fonction est, dans les minutes qui suivent, soulignée par deux brefs flashback, qui sont chaque fois raccordés dans le montage à partir d'un plan sur les yeux de la vieille dame: lorsqu'elle voit une image d'une porte du navire, enregistrée par une caméra sous-marine, puis le portrait que Jack (Leonardo DiCaprio) avait fait d'elle à l'époque, deux visions, de la porte en son état originel et de Jack en train de la dessiner, sont fugitivement insérées dans le film. Ses yeux voient littéralement le passé et sa mémoire s'annonce dès lors comme le moteur de la reconstitution. Après quelques échanges dilatoires autour d'un film en images de synthèse du naufrage, que commente un acolyte de Lovett en des termes brutaux et "chirurgicaux» («forensic»), selon le mot de Rose, celle-ci, devant un auditoire enfin disposé à l'écouter, peut lancer la reconstitution cinématographique. Derrière son visage en amorce, on aperçoit la proue du navire sur un moniteur. Le mouvement de la caméra vers la droite, qui laisse Rose hors 
champ, en position de narratrice, se recentre sur le Titanic en avançant vers lui, tandis que, par un trucage numérique, le paquebot se reconstitue progressivement dans ce qui semble être un seul et même plan.

Comme le remarque Peter Krämer (1999, p. 119), en reprenant et en amplifiant :

[...] les différents procédés narratifs (tels que les séquences oniriques, les voix off et les prologues autour de l'enfance) utilisés par les films d'action centrés sur des femmes [...] de façon à présenter l'action comme une partie ou une extension de la subjectivité du personnage principal féminin, Titanic présente résolument la majorité de son action comme une histoire racontée par une femme qui invite son auditoire, à l'écran et dans la salle, à partager ses souvenirs et donc à pénétrer son esprit.

Titanic est, dans le cycle des années 1990, le film qui va le plus loin dans cette féminisation du récit et sans doute aussi dans la conquête du public féminin (nous disposons de peu d'informations comparatives sur ce point, mais une enquête de l'hebdomadaire Newsweek indique que, pour Titanic, le public féminin était nettement plus nombreux que le public masculin, puisque $60 \%$ de la totalité des places de cinéma vendues aux États-Unis l'auraient été à des femmes ${ }^{11}$ ). Cependant, le processus ici décrit trouve à s'appliquer, à un moindre degré, dans des films comme Twister ou Deep Impact, qui favorisent l'empathie des spectateurs avec un personnage féminin, dont les émotions servent de fil conducteur au récit.

Ces éléments indiquent sans aucun doute que les studios hollywoodiens ont affiné leur stratégie commerciale, par rapport à une première époque du New Hollywood, les années 1970, où les goûts des adolescents et jeunes hommes ont été systématiquement privilégiés dès lors qu'il s'agissait de définir le public ciblé par les superproductions, le public féminin étant considéré «soit comme une niche commerciale, soit comme un complément au public jeune et masculin ${ }^{12}{ }^{\prime}$ (Krämer 1999, p. 124). Cet ajustement est, selon Peter Krämer, une façon de renouer avec l'époque classique, pendant laquelle les femmes, particulièrement les femmes d'âge mûr, étaient considérées comme les principales décisionnaires du programme cinématographique de 
la famille tout entière, et donc comme un "public clé» («key audience»), dont on avait intérêt à satisfaire les attentes si l'on voulait attirer le plus grand nombre de spectateurs possible (p. 125). L'hypothèse de l'auteur au sujet de Titanic, que j'étends volontiers au cycle des années 1990 dans son ensemble, est que l'amplification de la tonalité pathétique dans des films principalement orientés vers la présentation d'actions spectaculaires est une façon de remédier à une négligence commerciale, qui pourrait expliquer, notamment, la désaffection dont fut victime le film catastrophe à la fin des années 1970 .

\section{Perturbation/régulation}

Cette réorientation produit du "texte incohérent" (selon la fameuse expression de Robin Wood [2003, p. 41-62]), sur le plan générique et sur le plan des représentations genrées, puisque ces femmes agissantes mais malgré tout dominées - ou dominées mais malgré tout agissantes - font osciller les films entre discours progressiste et stéréotypes conservateurs. Comme je le signalais en introduction, il n'est pas simple d'assigner à un film un positionnement idéologique à partir du moment où l'on considère que celui-ci est fonction de la manière dont le texte est interprété: les cultural studies nous invitent ainsi à considérer que le public entre en relation avec le film à partir d'un certain nombre de compétences et de savoirs culturels déjà acquis, qui constituent des communautés interprétatives spécifiques et déterminent des postures d'acceptation, de négociation ou de résistance par rapport à une lecture préférentielle que le film sollicite et construit ${ }^{13}$.

Dans un essai dont le champ d'investigation dépasse largement celui du cinéma, Sue Thornham (2007, p. 67) propose quant à elle de distinguer entre potentiel perturbateur (disruptive potential) et stratégies de régulation (strategies of contain$m e n t$ ), qui peuvent cohabiter à l'intérieur d'un même texte et à partir desquels on peut s'efforcer de comprendre l'incohérence apparente d'un produit culturel donné. Je voudrais reprendre cette distinction en lui ajoutant deux variables: la structure $d u$ récit, soit l'intelligibilité du film à partir de l'agencement des événements racontés, et la puissance figurale, soit la composition 
plastique des plans perçus/mémorisés comme suites d'images indépendantes, jusqu'à un certain point, de la structure narrative dans laquelle elles s'insèrent ${ }^{14}$. Bien souvent, l'incohérence filmique provient d'un conflit entre ces deux éléments : la figure dérange, le récit recadre. Mais il est tout à fait possible d'imaginer des cas contraires, dans lesquels c'est la figure qui est stéréotypée, et le récit qui nous emmène là où il n'a pas l'habitude d'aller $^{15}$. Ou encore des cas où cohabitent, dans le même texte, figurations conservatrices et figurations subversives. En ce qui concerne les films catastrophe des années 1990, il est possible d'observer soit des films dans lesquels puissance figurale et structure du récit coïncident globalement, autour d'une légitimation $\mathrm{du}$ pouvoir patriarcal (je dirai donc de ces textes qu'ils sont a priori conservateurs, même s'il reste possible de les interpréter autrement), soit des films dans lesquels la figure est parfois perturbatrice et le récit toujours régulateur, à quelques détails près : dans ce cas, le texte peut donner lieu à des interprétations contradictoires, selon les éléments sélectionnés.

Cité par Mary Ann Doane (1987, p. 37) dans son essai sur le woman's film des années 1940, un dirigeant de l'International Division of the Motion Picture Association estimait en 1947 que :

Le film est un produit qui n'est jamais complètement consommé pour la bonne raison qu'il n'est jamais entièrement oublié par ceux qui l'ont vu. Il laisse derrière lui un résidu, un dépôt d'images et d'associations d'idées, ce qui en fait un produit unique dans notre formidable catalogue d'objets exportables.

Mais de quoi est fait ce résidu imaginaire? Que retient-on d'un film? Se souvient-on, dans Twister, du tempérament impétueux de Jo, qui harangue ses hommes avant d'aller affronter des tornades, et qui conduit à pleine vitesse un $4 \times 4$ flamboyant, ou bien de sa marginalisation scénaristique finale, quand son partenaire masculin reprend de façon prévisible le monopole de l'héroïsme? Lorsque le scénario est convenu et que l'image ne l'est pas, il est possible que ce soit la perturbation qui l'emporte sur la régulation, quand bien même celle-ci intervient en dernier, car on peut avancer l'hypothèse que ce qui dérange nos 
habitudes perceptives est aussi ce qui peut retenir l'attention ou frapper durablement les esprits. En proposant des images déréglées par rapport aux représentations traditionnelles des normes sexuées, le film catastrophe peut s'inscrire dans une telle logique. Ainsi, si l'on devait examiner de près le Titanic de James Cameron, on y trouverait sans aucun doute des figurations conservatrices des rapports homme-femme, dans lesquelles la femme n'est là que pour maintenir le love interest de l'homme, celui-ci apportant la preuve absolue de son héroïsme dans un acte chevaleresque ultime où il se sacrifie afin qu'elle puisse être sauvée. Le film offre même un exemple d'école du "plaisir visuel» essentiellement patriarcal mis en place par le cinéma hollywoodien, selon Laura Mulvey (1993, p. 18) ${ }^{16}$ : sous l'œil de Jack, Rose s'exhibe, se dénude et devient l'objet d'un regard masculin qui la détaille pendant qu'il la dessine, la réduisant à un "corps en fragments». Pourtant, cohabite avec cette représentation conventionnelle une séquence où Rose, hache à la main, brave l'eau glacée pour retrouver l'homme qu'elle aime et le sauver d'une noyade imminente. Cette figuration m'est proprement troublante, c'est-à-dire qu'elle dérange ma perception en refusant la régulation des représentations conventionnelles, et toujours dominantes aujourd'hui, d'une femme placée dans une situation d'extrême danger physique (femme passive ou apeurée), représentations renforcées par l'imagination elle aussi convenue d'un début de vingtième siècle corseté où les femmes étaient encore plus soumises aux injonctions patriarcales et confinées dans des identités sociales aliénantes. La singularité figurale de la séquence ouvre donc la voie à une interprétation progressiste du film dans son ensemble: de femme désirable, nue, allongée, chosifiée dans le regard de son amant qui la dessine, Rose devient ici femme agissante, dont le désir réinvestit les codes du film d'action. Le travelling arrière qui la montre, avançant vers la caméra, le corps au deux tiers plongé dans l'eau, son arme brandie au-dessus de ses épaules, propose une image de puissance qui contraste avec celles de l'«ancienne" Rose, étouffée par les convenances dues à son rang et à son sexe. Et cependant, la séquence est en même temps travaillée par une stratégie de régulation comique, dans la mesure où l'empowerment 
physique du personnage féminin est relativisé par la maladresse dont elle fait preuve, maladresse toute "féminine" qui réactive un cadre d'interprétation hétéronormatif de l'action. Rose doit briser les menottes de Jack avec sa hache mais ne parvient pas à frapper deux fois au même endroit et risque donc de blesser son amant en voulant le libérer. Le passage où elle s'entraîne contre une armoire en bois, sur la recommandation de Jack, expose de manière humoristique son défaut de coordination. Jack fait mine de trouver l'essai concluant et l'assure qu'elle va très bien s'en tirer, non sans lui prodiguer d'ultimes conseils. Devant l'inaptitude de son "élève", l'homme, ici en position de formateur, s'en remet à un acte de foi. C'est d'ailleurs les yeux fermés que Rose abattra sa hache sur les menottes de Jack: la libération a bien lieu, le personnage féminin en est bien l'agent, mais son courage et sa détermination n'ont pas mis un terme à son déficit de compétence, et c'est la chance, ainsi peut-être que le discours de Jack l'assurant de sa pleine confiance, qui permettent la libération. Dès lors, comment interpréter la séquence? Faut-il retenir la prise d'initiative du personnage féminin, d'ailleurs confronté tout au long de l'épisode à des hommes paniqués, incapables de lui venir en aide, l'un d'eux finissant, pour sa lâcheté, par recevoir de sa part un coup de poing en plein visage? Ou bien doit-on s'arrêter aux limites imposées à cette figuration de l'action woman, en remarquant que, de manière conventionnelle, c'est Jack qui la guide ou l'inspire, et que la scène met en lumière l'inadaptation foncière de la femme au registre de l'action physique, qu'elle ne peut investir que temporairement et sur un mode comique?

Lorsque Rose brave les dangers que tous les hommes fuient, lorsque la force physique d'Amy est sollicitée pour aider un groupe d'hommes à déplacer un bus dans la fournaise de l'éruption (Volcano) ou lorsque Jo retrouve la posture protectrice du corps masculin pour protéger un de ses collaborateurs blessés (Twister), le film catastrophe propose des images d'un pouvoir féminin qui s'exerce sur le terrain de compétence traditionnel de la masculinité hérö̈que (courage, performances athlétiques). Mais que faut-il faire de ces images, par ailleurs contestées par des structures narratives qui accordent une place de choix à 


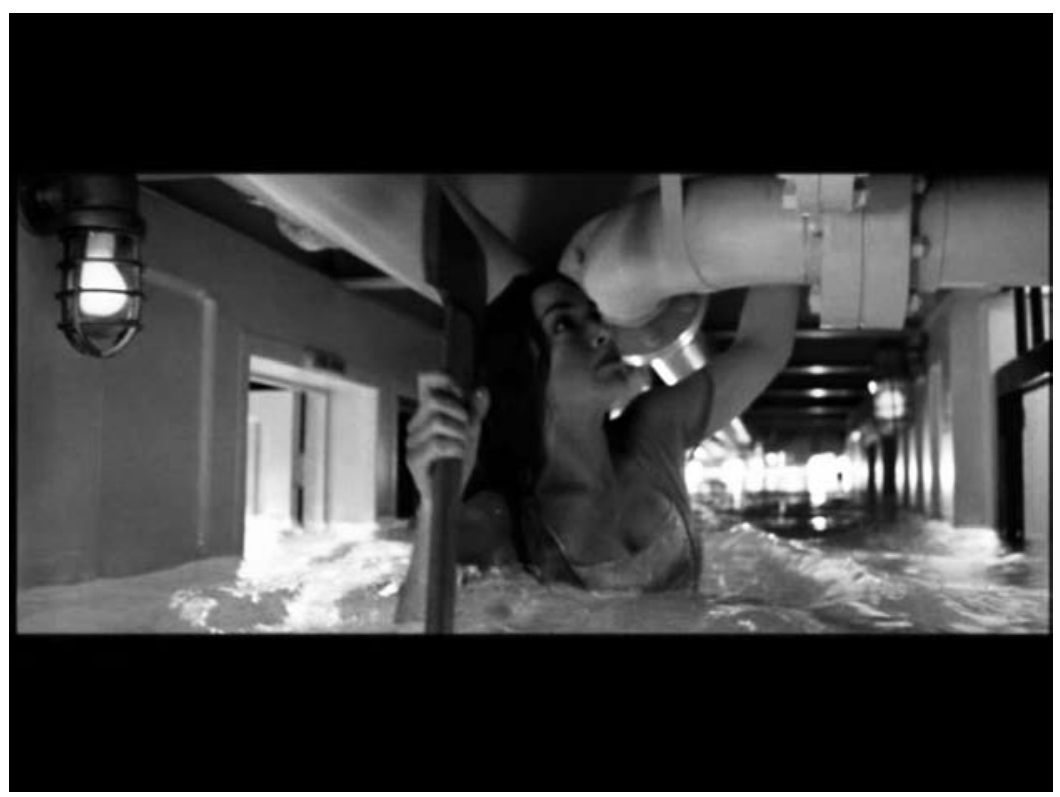

Titanic (James Cameron, 1997). C Twentieth Century Fox et Paramount Pictures.

l'héroïsme masculin et valident une représentation stéréotypée des rapports de sexe? Peut-on considérer qu'elles participent malgré tout, dans le cadre d'un cinéma populaire saturé de clichés, à la remise en question du traditionnel leadership masculin, donc à la déstabilisation des stéréotypes? Mon sentiment est qu'elles en offrent la possibilité, mais que c'est le spectateur (la spectatrice) qui actualisera, ou pas, cette interprétation. L'ambiguïté du progressisme hollywoodien se trouve ici posée et reste ouverte au débat, comme ce fut le cas pour Thelma \& Louise au moment de sa sortie. À propos de ce film, Yvonne Tasker (1993, p. 95) écrivait :

Une critique idéologique basée sur le contenu des films est, à beaucoup d'égards, incompatible avec une compréhension du cinéma comme fantasme, comme expérience sensuelle, comme une série de moments raccordés par un récit.

L'expérience des images peut défaire le discours du récit, la transgression des normes persister malgré le retour à l'ordre. Rose est peut-être devenue une vieille potière, assagie et 


\title{
domestiquée: Titanic n'en rend pas moins mémorable l'indé- pendance et la bravoure de la jeune femme qu'elle fut.
}

\author{
Université de Poitiers
}

\section{NOTES}

1. De telles analyses sont par exemple développées par Michael Ryan et Douglas Kellner (1988, p. 52-57). En France, Ignacio Ramonet (1980, p. 19-39) a également proposé une lecture idéologique similaire.

2. "The frights, like those from "Earthquake" and "Towering Inferno", are not put away by happy endings». Sauf mention contraire, toutes les traductions de l'anglais sont de moi.

3. L'objet de cet article n'étant pas de s'appesantir sur la définition problématique du genre, j'indique simplement que, à partir d'une analyse sémantico-syntaxique inspirée des travaux de Rick Altman, je redéfinis les films catastrophe comme des films où la catastrophe occupe une place centrale d'un point de vue narratif, esthétique et idéologique, et qui représentent une communauté en crise mais finalement purgée de ses maux, dans un récit à vocation prioritairement spectaculaire. Le genre se constitue au début des années 1970, date à laquelle se répand l'étiquette générique disaster film ou disaster movie, à partir des succès commerciaux obtenus par des films comme L'aventure $d u$ Poséidon ou La tour infernale. Après de fracassants échecs à la fin de la décennie et une éclipse au cours des années 1980, le genre fait un retour dans les années 1990, et c'est de ce deuxième cycle que le présent article traite en particulier. Pour un examen approfondi du film catastrophe en tant que genre, voir Keane 2001 et Molia 2007.

4. C'est cette vision qui donne par exemple sa forme à l'essai de Robin Wood, dont le titre est en soi évocateur: Hollywood from Vietnam to Reagan... and Beyond (2003).

5. J'explique plus loin ce que cette formule désigne pour moi.

6. «It is man's work and you are just little girlies. I can't let you go down there».

7. Sur ce stéréotype, on pourra consulter Yvonne Tasker (1993a, p. 132-152) et Raphaëlle Moine (2010, p. 108-113).

8. http://www.lesbilicious.co.uk/tv-film/linda-hamilton-in-new-terminator-film/.

9. Pour un aperçu des études des goûts du public aux États-Unis, on pourra se reporter au travail de Geoff King (2002, p. 197-198) sur le film d'action, qui s’appuie sur le rapport 1999 Motion Picture Attendance. En ce qui concerne la France, les travaux de Jean-Michel Guy se révèlent éclairants. Ses études statistiques établissent que "[l]es hommes montrent ainsi une prédilection pour les genres "violents" (films violents, films de karaté, de guerre, etc.) ou faisant souvent la part belle à l'action violente comme la science-fiction, le western, l'horreur ou certaines formes de l'érotisme. Laction en général plait à un plus grand nombre d'hommes que de femmes, mais elle suscite moins d'opposition que ses déclinaisons explicitement violentes. Même l'aventure est une catégorie qui réunit plus d'amateurs masculins, ce qui laisse entendre que les films d'aventure comportent une part de violence qui rebute les femmes. Les femmes sont, en comparaison, beaucoup plus portées sur les films sentimentaux, les comédies musicales, les films d'opéra ou de musique classique, les films psychologiques et les films dits "d'auteur" " (Guy 2000, p. 119). Cinquante pour cent (50\%) des femmes françaises disent aimer les films d'action contre $69 \%$ des hommes; les films catastrophe (mais il faudrait savoir quels films sont désignés par là) sont appréciés par $28 \%$ des hommes et $21 \%$ des femmes. 
10. "This thing is not over. We've just seen the beginning!"

11. Enquête citée par Peter Krämer (1999, p. 124).

12. «[...] either a niche market or an adjunct to the young male audience».

13. Dans un article fondateur, Stuart Hall (1980) propose de distinguer ces trois modes d'appropriation: dominant, negotiated, oppositional.

14. Il faut noter que la stratégie commerciale des studios hollywoodiens accentue la désaffiliation des images par rapport à la structure narrative, dans la mesure où la communication et le marketing autour du film tendent à lui substituer des images fortes censées le contenir tout entier. Cette équation un film = une image (ou une série limitée d'images) est construite et martelée par les dossiers de presse, les bandesannonces et les teasers, les affiches et autres supports promotionnels. Une recherche dans Google images sur un film donne aujourd'hui à voir un tel phénomène de réduction figurale, les mêmes deux ou trois images se retrouvant sur des dizaines voire des centaines de sites différents. Aliens (James Cameron, 1986) se résume de la sorte à l'image de Ripley tenant une fillette dans un bras et une mitraillette dans l'autre, Thelma \& Louise (Ridley Scott, 1991) donne à voir deux femmes à l'accoutrement rebelle appuyées contre le capot d'une voiture, etc.

15. Les parodies offrent de tels exemples: la reprise iconographique d'un genre donné y est subvertie par sa mise en récit comique.

16. Des extraits de son fameux article "Visual Pleasure and Narrative Cinema", paru dans Screen à l'automne 1975, sont accessibles en français dans le numéro de CinémAction intitulé 20 ans de théories féministes sur le cinéma: Grande-Bretagne et États-Unis (Mulvey 1993).

\section{RÉFÉRENCES BIBLIOGRAPHIQUES}

Champlin 1975: Charles Champlin, "Don't Go Near the Water », LA Times, 20 juin 1975 , p. 35.

Doane 1987: Mary Ann Doane, The Desire to Desire. The Woman's Film of the 1940s, Bloomington/Indianapolis, Indiana University Press, 1987.

Guy 2000 : Jean-Michel Guy, La culture cinématographique des Français, Paris, La Documentation Française, 2000.

Hall 1980 : Stuart Hall, "Encoding/Decoding», dans Stuart Hall, Dorothy Hobson, Andrew Lowe et Paul Willis (dir.), Culture, Media, Language. Working Papers in Cultural Studies, London, Unwin Hyman, 1980, p. 128-138.

Keane 2001 : Stephen Keane, Disaster Movies. The Cinema of Catastrophe, London/New York, Wallflower, 2001.

King 2002 : Geoff King, New Hollywood Cinema. An Introduction, London/ New York, I.B. Tauris Publishers, 2002.

Krämer 1999: Peter Krämer, "Women First. Titanic, Action-Adventure Films, and Hollywood's Female Audience», dans Kevin S. Sandlar et Gaylyn Studlar (dir.), Titanic. Anatomy of a Blockbuster, New Brunswick (N.-J.)/London, Rutgers University Press, 1999, p. 108-131.

Moine 2010 : Raphaëlle Moine, Les femmes d'action au cinéma, Paris, Armand Colin, 2010.

Molia 2007 : François-Xavier Molia, Un cinéma de la destruction. Approches esthétique, historique et industrielle du film-catastrophe hollywoodien, thèse de doctorat, Paris XNanterre, 2007.

Mulvey 1993: Laura Mulvey, «Plaisir visuel et cinéma narratif», traduit de l'anglais par Valérie Hébert et Bérénice Reynaud, CinémAction, nº 67, 1993, p. 17-23. 
Ramonet 1980 : Ignacio Ramonet, "Les "films-catastrophes" américains. Des fictions pour la crise", Le chewing-gum des yeux, Paris, Alain Moreau, 1980, p. 19-39.

Ryan et Kellner 1988 : Michael Ryan et Douglas Kellner, Camera Politica. The Politics and Ideology of Contemporary Hollywood Film, Bloomington/Indianapolis, Indiana University Press, 1988.

Tasker 1993: Yvonne Tasker, "Criminelles: Thelma et Louise et autres délinquantes", traduit de l'anglais par Ginette Vincendeau, CinémAction, n 67, 1993, p. 95.

Tasker 1993a: Yvonne Tasker, Spectacular Bodies. Gender, Genre and the Action Cinema, London, Routledge, 1993.

Thornham 2007 : Sue Thornham, Women, Feminism and Media, Edimbourg, Edinburgh University Press, 2007.

Wood 2003: Robin Wood, Hollywood from Vietnam to Reagan... and Beyond [1986], Expanded and Revised Edition, New York, Columbia University Press, 2003.

\section{ABSTRACT}

\section{"It is man's work and you are all just little girlies": Gendered Narration and Figures of Female Empowerment in the Hollywood Disaster Film François-Xavier Molia}

The Hollywood disaster film of the 1970s reproduced traditional representations of sexual relations in a patriarchal society. In the 1990s, this genre appeared to reflect the evolution of contemporary society by granting women a more prominent presence on the screen, as well as greater social status. What was the possible outcome, with respect to the evolution of the genre, of this re-equilibrium between film characters? Twister (Jan de Bont, 1996), Deep Impact (Mimi Leder, 1998) and Titanic (James Cameron, 1997) modified the disaster film formula by grafting onto it elements which could (at least one supposes) attract female viewers to a "men's genre": in-depth treatment of romantic and family situations; crossovers with the melodrama and the romance film and amplification of their pathetic tone; and, finally, exploration of the female point of view using a variety of narrative techniques. This reorientation created an "incoherent text" (Robin Wood) on the level of genre and that of gender representations, because these active yet ultimately dominated women caused the films to waver between progressive vision and stereotypes. 